\title{
Modelo Matemático para Descrever Transmissão de Leishmaniose $^{1}$
}

J.C. ROSALES ${ }^{2}$, Programa de Pós-graduação em Matemática Aplicada, IMECC, UNICAMP e Lab-Epifisma, Caixa Postal 6065, CEP 13081-970, Campinas, SP, Brasil.

H.M. YANG ${ }^{3}$, Departamento de Matemática Aplicada, IMECC, UNICAMP e Lab-Epifisma, Cx.P. 6065, 13081-970, Campinas, SP, Brasil.

Resumo: Analisa-se um modelo compartimental para a transmisão de Leishmaniose que contempla o homem, cão e Lutzomyia como hospedeiros vertebrados e invertebrado, respetivamente. Deriva-se a expressão do número de reprodutibilidade basal. Estuda-se a estabilidade. Aplica-se o mesmo para simular a situação de zonas endêmicas no Estado de São Paulo.

\section{Introdução}

A Leishmaniose é uma doença amplamente extendida em 22 países do Novo Mundo e 66 do Velho Mundo. A Leishmaniose Visceral (LV) é geralmente fatal quando não é tratada. A Leishmaniose Muco-Cutânea (LMC) é uma doença mutilante que pode chegar a metástasis ou lesões incapacitantes. A Leishmaniose Cutânea (LC) e, também, o caso da Leishmaniose Cutânea Difusa (LCD) são outras de suas manifestações [6] [7].

A incidência anual desta doença é estimada em 1-1,5 milhões de casos de LC e 0,5 milhões de casos de LV, no Velho Mundo. A incidência total no mundo é de 12 milhões de pessoas e a população total em risco ronda a casa dos 350 milhões de pessoas [7]. Por causa desses números a Organização Mundial de Saúde (OMS) considera esta entre as seis mais importantes doenças do mundo. Em 1993, a OMS considerou Leishmaniose como a segunda doença causada por protozoários de importância em saúde pública.

Esta doença é de caráter zoonótico que acomete o homem e diversos animais domésticos e silvestres. A Leishmaniose é causada por parasitas do gênero Leishmania Ross, um protozoário digenético que tem seu ciclo biológico composto de hospedeiros vertebrados e invertebrados. Os hospedeiros invertebrados são pequenos insetos da ordem Diptera, família Psychodydade, subfamilia Phlebotominae, gênero Lutzomyia [4].

\footnotetext{
${ }^{1}$ Apoio financeiro FAPESP (Projeto Temático) e CNPq (Edital Universal).

${ }^{2}$ Bolsista de BID-Japan Scholarship Program (jcrsalta@yahoo.com.ar).

${ }^{3}$ Bolsista CNPq (hyunyang@ime.unicamp.br).
} 
Nesse trabalho propomos um modelo matemático para descrever a transmissão de Leishmaniose na população humana. Entretanto, a ocorrência da LV em seres humanos tem como fator de risco a transmissão de LV em cães com prevalência superior a $2 \%$, em conjunção com uma alta densidade de população canina [1]. Portanto, acopla-se a população canina no modelo, além do vetor flebotomíneo. Na seção 2 o modelo é apresentado, com uma aplicação numérica na seção 3 . Conclusão é dada na seção 4 .

\section{Modelagem Matemática}

Desenvolvendo um modelo matemático, estuda-se a transmissão urbana de Leishmaniose, onde o cão é o hospedeiro definitivo, enquanto o homem é ocasional. O estudo da dinâmica nesse caso inclui os hospedeiros vertebrados (o homem e o cão) e invertebrado (o flebotomíneo).

\subsection{Modelo Matemático}

A transmissão de parasitas do gênero Leishmania Ross é descrita quantitativamente assumindo que a infecção ocorre entre as várias populações conforme a lei da ação das massas. O modelo não leva em consideração os períodos de incubação do parasita nem nos hospedeiros, nem no vetor. Assume-se que os hospedeiros vertebrados desenvolvem uma resposta imunológica parcial, porém o vetor flebotomíneo é incapaz de responder imunologicamente. Assim, os vertebrados são capazes de infectar insetos durante o período infeccioso, enquanto os vetores, quando infectados, transmitem o protozoário aos vertebrados durante todo o seu período de vida.

Assim, a interação entre vertebrados e inseto é analisada considerando, para os humanos, três compartimentos que representam as frações de indivíduos no tempo $t$, de suscetível $H_{1}(t)$, infectado $H_{2}(t)$ e recuperado $H_{3}(t)$. Para os cães, similarmente, é dividida em frações de suscetível $C_{1}(t)$, infectado $C_{2}(t)$ e recuperado $C_{3}(t)$. A população do vetor flebotomíneos é dividida só em frações de suscetível $L_{1}(t)$ e infectado $L_{2}(t)$. A interação entre os vertebrados e os vetores flebotomíneos pode ser descrito por um sistema de equações diferenciais ordinárias não lineares,

$$
\left\{\begin{array}{l}
\frac{d H_{1}(t)}{d t}=\phi_{1}+\theta_{1} H_{3}(t)-\left(\alpha_{1} L_{2}(t)+\mu_{1}\right) H_{1}(t) \\
\frac{d H_{2}(t)}{d t}=\alpha_{1} L_{2}(t) H_{1}(t)-\left(\mu_{1}+\mu_{1}^{\prime}+\gamma_{1}\right) H_{2}(t) \\
\frac{d H_{3}(t)}{d t}=\gamma_{1} H_{2}(t)-\left(\mu_{1}+\theta_{1}\right) H_{3}(t) \\
\frac{d C_{1}(t)}{d t}=\phi_{2}+\theta_{2} C_{3}(t)-\left(\alpha_{2} L_{2}(t)+\mu_{2}\right) C_{1}(t) \\
\frac{d C_{2}(t)}{d t}=\alpha_{2} L_{2}(t) C_{1}(t)-\left(\mu_{2}+\mu_{2}^{\prime}+\gamma_{2}\right) C_{2}(t) \\
\frac{d C_{3}(t)}{d t}=\gamma_{2} C_{2}(t)-\left(\mu_{2}+\theta_{2}\right) C_{3}(t) \\
\frac{d L_{1}(t)}{d t}=\phi_{3}-\left(\beta_{1} H_{2}(t)+\beta_{2} C_{2}(t)+\mu_{3}\right) L_{1}(t) \\
\frac{d L_{2}(t)}{d t}=\left(\beta_{1} H_{2}(t)+\beta_{2} C_{2}(t)\right) L_{1}(t)-\left(\mu_{3}+\mu_{3}^{\prime}\right) L_{2}(t),
\end{array}\right.
$$

onde $\mu_{i}$ e $\mu_{i}^{\prime}$ são, respectivamente, as taxas de mortalidade natural e induzida pela doença, $\alpha_{i}$ e $\beta_{i}$ são as taxas de contato entre vertebrados e insetos, $\theta_{i}$ é a taxa de perda de imunidade, $\gamma_{i}^{-1}$ é o período de recuperação ou infeccioso. O índice $i, i=1$, 
2 e 3, representa população de, respectivamente, humanos $(H)$, cães $(C)$ e insetos $(L)$. Todos os parâmetros são positivos.

O sistema de equações (2.1) assume que populações envolvidas são constantes, o que permite normalizá-las de modo simples, ou,

$$
\sum_{i=1}^{3} H_{i}=1 \quad \sum_{i=1}^{3} C_{i}=1 \quad \sum_{i=1}^{2} L_{i}=1
$$

Ainda, assume-se que nesta comunidade não ocorrem migrações, ou

$$
\sum_{i=1}^{3} \frac{d H_{i}(t)}{d t}=0 \quad \sum_{i=1}^{3} \frac{d C_{i}(t)}{d t}=0 \quad \sum_{i=1}^{2} \frac{d L_{i}(t)}{d t}=0 .
$$

As duas suposições resultam para as funções de entrada $\phi_{i}, i=1,2$ e 3 , as relações

$$
\phi_{1}=\mu_{1}+\mu_{1}^{\prime} H_{2}(t) \quad \phi_{2}=\mu_{2}+\mu_{2}^{\prime} C_{2}(t) \quad \phi_{3}=\mu_{3}+\mu_{3}^{\prime} L_{2}(t) .
$$

Essas relações mostram que os nascimentos compensam as mortes por causas naturais e devido a doença.

Em seguida, determina-se os pontos de equilíbrio e a estabilidade desses pontos.

\subsection{Análise do Modelo}

A análise do modelo visa obter o número de reprodutibilidade basal, denotado por $R_{0}$, que é um conceito de suma importância na epidemiologia matemática. A expressão que se obtém do modelo para $R_{0}$ fornece uma importante informação acerca das possíveis ações para manter a doença sob controle. Como vamos mostrar, se $R_{0}>$ 1, então teremos níveis endêmicos da doença, ou seja, a doença se estabelecerá na população, enquanto para $R_{0}<1$, a população de infectados diminuirá, até desaparecer.

\subsubsection{Ponto de Equilíbrio Trivial}

Em primeiro lugar, mostra-se que existe um ponto de equilíbrio trivial $\overline{P_{e_{1}}}$, que descreve uma comunidade livre da doença, com as coordenadas

$$
\left\{\begin{array} { l } 
{ H _ { 1 } = 1 } \\
{ H _ { 2 } = H _ { 3 } = 0 }
\end{array} \quad \left\{\begin{array} { l } 
{ C _ { 1 } = 1 } \\
{ C _ { 2 } = C _ { 3 } = 0 }
\end{array} \quad \left\{\begin{array}{l}
L_{1}=1 \\
L_{2}=0 .
\end{array}\right.\right.\right.
$$

A estabilidade do ponto de equilíbrio pode ser determinada pelas raízes do polinômio característico $P(\lambda)$. O mesmo é definido por $P(\lambda)=\operatorname{det}(J-\lambda I)$, onde $J$ é o Jacobiano, formado pelas derivadas parciais em relação às variáveis de estado das funções que essão no segundo membro do sistema (2.1), calculado no ponto de equilíbrio $\overline{P_{e_{1}}}$. Neste caso temos o seguinte resultado:

Teorema 2.1. O ponto de equilíbrio trivial $\overline{P_{e_{1}}}$ do sistema (2.1) é localmente e assintoticamente estável se o número de reprodutibilidade basal for $R_{0}<1$, onde $R_{0} e ́$ definido como $R_{0}=\frac{\beta_{1}}{\beta_{1}^{0}}+\frac{\beta_{2}}{\beta_{2}^{0}}, \operatorname{com} \beta_{1}^{0}=\frac{\left(\mu_{1}+\mu_{1}^{\prime}+\gamma_{1}\right)\left(\mu_{3}+\mu_{3}^{\prime}\right)}{\alpha_{1}}$ e $\beta_{2}^{0}=\frac{\left(\mu_{2}+\mu_{2}^{\prime}+\gamma_{2}\right)\left(\mu_{3}+\mu_{3}^{\prime}\right)}{\alpha_{2}}$. 
Demonstração. Para o ponto de equilíbrio $\overline{P_{e_{1}}}$, correspondente à comunidade livre de doença, a expressão do polinômio característico $P(\lambda)$ é

$$
P(\lambda)=\left(\mu_{3}+\lambda\right) \prod_{i=1}^{2}\left(\mu_{i}+\lambda\right)\left(\mu_{i}+\theta_{i}+\lambda\right) P_{3}(\lambda)
$$

com o último polinômio de terceiro grau dado por

$$
P_{3}(\lambda)=\left(\overline{\mu_{1}}+\lambda\right)\left[\left(\overline{\mu_{2}}+\lambda\right)\left(\overline{\mu_{L}}+\lambda\right)-\alpha_{2} \beta_{2}\right]-\alpha_{1} \beta_{1}\left(\overline{\mu_{2}}+\lambda\right),
$$

onde as mortalidades generalizadas são dadas por

$$
\left\{\begin{array}{l}
\overline{\mu_{1}}=\mu_{1}+\mu_{1}^{\prime}+\gamma_{1} \\
\overline{\mu_{2}}=\mu_{2}+\mu_{2}^{\prime}+\gamma_{2} \\
\overline{\mu_{L}}=\mu_{3}+\mu_{3}^{\prime}
\end{array}\right.
$$

Primeiro, tem-se os autovalores $\lambda_{1}=-\mu_{1}, \lambda_{2}=-\mu_{2}, \lambda_{3}=-\mu_{3}, \lambda_{4}=-\left(\mu_{1}+\theta_{1}\right)$ e $\lambda_{5}=-\left(\mu_{2}+\theta_{2}\right)$, que são negativos, pois os parâmetros são positivos. Só falta determinar os três autovalores restantes, designados por $\lambda_{6}, \lambda_{7}$ e $\lambda_{8}$, raízes de $P_{3}(\lambda)$. Como é um polinômio de terceiro grau, determina-se o sinal dos autovalores $\lambda_{6}, \lambda_{7}$ e $\lambda_{8}$. Para tal, usamos os critérios de Routh-Hurwitz [2] [5], que estabelece critérios a respeito do sinal das raízes: se $a_{1}>0, a_{3}>0$ e $a_{1} a_{2}>a_{3}$, então todas as raízes tem parte real negativa. O polinômio de terceiro grau $P_{3}(\lambda)$ é re-escrito como

$$
P_{3}(\lambda)=\lambda^{3}+a_{1} \lambda^{2}+a_{2} \lambda+a_{3}
$$

com os coeficientes dados por

$$
\left\{\begin{array}{l}
a_{1}=\overline{\mu_{1}}+\overline{\mu_{2}}+\overline{\mu_{L}} \\
a_{2}=\overline{\mu_{1}}\left[\overline{\mu_{2}}+\overline{\mu_{L}}\right]+\overline{\mu_{2} \mu_{L}}-\left(\alpha_{1} \beta_{1}+\alpha_{2} \beta_{2}\right) \\
a_{3}=\overline{\mu_{1} \mu_{2} \mu_{L}}\left(1-R_{0}\right) .
\end{array}\right.
$$

O primeiro critério é satisfeito, pois é verdade que $a_{1}>0$. O segundo critério $a_{3}>0$ é satisfeito se $R_{0}<1$. Verifiquemos, agora, se satisfaz a última condição $a_{1} a_{2}>a_{3}$. Para isso, utiliza-se a condição necessária para satisfazer $a_{3}>0$. Assim, uma vez que $R_{0}<1$, então tem-se que $1-\frac{\beta_{1}}{\beta_{1}^{0}}-\frac{\beta_{2}}{\beta_{2}^{0}}>0$, o que implica em $1-\frac{\beta_{1}}{\beta_{1}^{0}}>0$ e $1-\frac{\beta_{2}}{\beta_{2}^{0}}>0$. Logo, constroi-se a desigualdade

$$
\frac{\overline{\mu_{1}}+\overline{\mu_{2}}}{\overline{\mu_{L}}}+\left(\overline{\mu_{1}}+\overline{\mu_{2}}+\overline{\mu_{L}}\right)\left[\frac{1}{\overline{\mu_{2}}}\left(1-\frac{\beta_{1}}{\beta_{1}^{0}}\right)+\frac{1}{\overline{\mu_{1}}}\left(1-\frac{\beta_{2}}{\beta_{2}^{0}}\right)\right]+R_{0}>0,
$$

que, somando 1 nos dois membros, resulta em

$$
\frac{\overline{\mu_{1}}+\overline{\mu_{2}}}{\overline{\mu_{L}}}+1+\left(\overline{\mu_{1}}+\overline{\mu_{2}}+\overline{\mu_{L}}\right)\left[\frac{1}{\overline{\mu_{2}}}\left(1-\frac{\beta_{1}}{\beta_{1}^{0}}\right)+\frac{1}{\overline{\mu_{1}}}\left(1-\frac{\beta_{2}}{\beta_{2}^{0}}\right)\right]+R_{0}>1 .
$$

Agora, subtraindo $R_{0}$ nos dois membros, tem-se

$$
\frac{\overline{\mu_{1}}+\overline{\mu_{2}}+\overline{\mu_{L}}}{\overline{\mu_{L}}}+\left(\overline{\mu_{1}}+\overline{\mu_{2}}+\overline{\mu_{L}}\right)\left[\frac{1}{\overline{\mu_{2}}}\left(1-\frac{\beta_{1}}{\beta_{1}^{0}}\right)+\frac{1}{\overline{\mu_{1}}}\left(1-\frac{\beta_{2}}{\beta_{2}^{0}}\right)\right]>1-R_{0},
$$


que, re-arranjada, torna-se

$$
\left(\overline{\mu_{1}}+\overline{\mu_{2}}+\overline{\mu_{L}}\right)\left[\frac{1}{\overline{\mu_{L}}}+\frac{1}{\overline{\mu_{2}}}\left(1-\frac{\beta_{1}}{\beta_{1}^{0}}\right)+\frac{1}{\overline{\mu_{1}}}\left(1-\frac{\beta_{2}}{\beta_{2}^{0}}\right)\right]>1-R_{0} .
$$

Finalmente, multiplicando por $\overline{\mu_{1} \mu_{2} \mu_{L}}>0$ nos dois membros, obtem-se a hipótese proposta $a_{1} a_{2}>a_{3}$.

Resumindo, o ponto de equilibrio trivial $\overline{P_{e}}$, em que a comunidade encontra-se livre da doença, é localmente e assintoticamente estável se $R_{0}<1$, pois satisfaz os critérios $a_{1}>0, a_{3}>0$ e $a_{1} a_{2}>a_{3}$. Note que uma vez satisfeito $a_{3}>0$, os outros critérios são automaticamente satisfeitos.

\subsubsection{Ponto de equilíbrio não trivial}

Analisamos o caso do equilíbrio não trivial, ou seja, a população apresenta níveis endêmicos de Leishmaniose. Para isso estudamos o sistema (2.1) em estado estacionário.

A fração de homens recuperados em equilíbrio é dada por

$$
H_{3}=\frac{\gamma_{1}}{\mu_{1}+\theta_{1}} H_{2}
$$

e a expressão correspondente para homens infectados resulta em

$$
H_{2}=\frac{\left(\mu_{1}+\theta_{1}\right) \alpha_{1} L_{2}}{\left(\mu_{1}+\theta_{1}+\gamma_{1}\right) \alpha_{1} L_{2}+\left(\mu_{1}+\theta_{1}\right)\left(\mu_{1}+\mu_{1}^{\prime}+\gamma_{1}\right)} .
$$

Analogamente, temos para o caso dos cães recuperados e infectados,

$$
C_{3}=\frac{\gamma_{2}}{\mu_{2}+\theta_{2}} C_{2}
$$

e

$$
C_{2}=\frac{\left(\mu_{2}+\theta_{2}\right) \alpha_{2} L_{2}}{\left(\mu_{2}+\theta_{2}+\gamma_{2}\right) \alpha_{2} L_{2}+\left(\mu_{2}+\theta_{2}\right)\left(\mu_{2}+\mu_{2}^{\prime}+\gamma_{2}\right)} .
$$

Usando as relações dadas na equação (2.2), pode-se facilmente obter as expressões para $H_{1}$ e $C_{1}$. Finalmente, temos as seguintes expressões para os hospedeiros invertebrados,

$$
L_{1}=\frac{\mu_{3}+\mu_{3}^{\prime} L_{2}}{\mu_{3}+\beta_{1} H_{2}+\beta_{2} C_{2}}
$$

$\mathrm{e}$

$$
L_{2}=\frac{\left(\beta_{1} H_{2}+\beta_{2} C_{2}\right)}{\mu_{3}+\mu_{3}^{\prime}} \frac{\left(\mu_{3}+\mu_{3}^{\prime} L_{2}\right)}{\mu_{3}+\beta_{1} H_{2}+\beta_{2} C_{2}},
$$

de onde resulta

$$
\mu_{3}\left(\mu_{3}+\mu_{3}^{\prime}\right) L_{2}+\mu_{3}\left(\beta_{1} H_{2}+\beta_{2} C_{2}\right) L_{2}-\mu_{3}\left(\beta_{1} H_{2}+\beta_{2} C_{2}\right)=0 .
$$


Para obter uma equação em termos de uma única variável de estado, subsitui-se as equações (2.5) e (2.6) na equação (2.7). O resultado é um polinômio de terceiro grau para $L_{2}$,

$$
P\left(L_{2}\right)=\left(b_{2} L_{2}^{2}+b_{1} L_{2}+b_{0}\right) L_{2},
$$

com os seguintes coeficientes

$$
\left\{\begin{aligned}
b_{2}= & \alpha_{1} \alpha_{2} \mu_{3}\left[\overline{\mu_{L}}\left(\mu_{1}+\theta_{1}+\gamma_{1}\right)\left(\mu_{2}+\theta_{2}+\gamma_{2}\right)+\left(\mu_{1}+\theta_{1}\right)\left(\mu_{2}+\theta_{2}+\gamma_{2}\right) \beta_{1}\right. \\
& \left.+\left(\mu_{2}+\theta_{2}\right)\left(\mu_{1}+\theta_{1}+\gamma_{1}\right) \beta_{2}\right] \\
b_{1}= & \mu_{3} \overline{\mu_{L}}\left[\left(\mu_{2}+\theta_{2}\right) \overline{\mu_{2}}\left(\mu_{1}+\theta_{1}+\gamma_{1}\right) \alpha_{1}+\left(\mu_{1}+\theta_{1}\right) \overline{\mu_{1}}\left(\mu_{2}+\theta_{2}+\gamma_{2}\right) \alpha_{2}\right] \\
& +\mu_{3}\left(\mu_{1}+\theta_{1}\right)\left(\mu_{2}+\theta_{2}\right)\left[\alpha_{1} \beta_{1} \overline{\mu_{2}}+\alpha_{2} \beta_{2} \overline{\mu_{1}}\right] \\
& -\mu_{3} \alpha_{1} \alpha_{2}\left[\left(\mu_{1}+\theta_{1}\right)\left(\mu_{2}+\theta_{2}+\gamma_{2}\right) \beta_{1}+\left(\mu_{2}+\theta_{2}\right)\left(\mu_{1}+\theta_{1}+\gamma_{1}\right) \beta_{2}\right] \\
b_{0}= & \mu_{3}\left(\mu_{1}+\theta_{1}\right)\left(\mu_{2}+\theta_{2}\right)\left[\overline{\mu_{L}} \overline{\mu_{1}} \overline{\mu_{2}}-\alpha_{1} \beta_{1} \overline{\mu_{2}}-\alpha_{2} \beta_{2} \overline{\mu_{1}}\right] .
\end{aligned}\right.
$$

Primeiro, vamos mostrar a unicidade de solução do polinômio de segundo grau, equação (2.8), aplicando a regra de Descartes [5]. Como tem-se $b_{2}>0$ sempre, basta analisar o sinal de $b_{0}$ e $b_{1}$. Para o coeficiente $b_{0}$, re-escrevemos em função do número de reprodutibilidade basal, $R_{0}$, ou

$$
b_{0}=\mu_{3}\left(\mu_{1}+\theta_{1}\right)\left(\mu_{2}+\theta_{2}\right) \overline{\mu_{L}} \overline{\mu_{1} \mu_{2}}\left(1-R_{0}\right),
$$

enquanto para $b_{1}$, agrupamos convenientemente para obter

$$
\begin{aligned}
b_{1}= & \mu_{3}\left(\mu_{1}+\theta_{1}\right) \overline{\mu_{L} \mu_{1}}\left(\mu_{2}+\theta_{2}+\gamma_{2}\right) \alpha_{2}\left(1-\frac{\beta_{1}}{\beta_{1}^{0}}\right) \\
& +\mu_{3}\left(\mu_{2}+\theta_{2}\right) \overline{\mu_{L} \mu_{2}}\left(\mu_{1}+\theta_{1}+\gamma_{1}\right) \alpha_{1}\left(1-\frac{\beta_{2}}{\beta_{2}^{0}}\right) \\
& +\mu_{3}\left(\mu_{1}+\theta_{1}\right)\left(\mu_{2}+\theta_{2}\right) \overline{\mu_{L} \mu_{1} \mu_{2}} R_{0} .
\end{aligned}
$$

Portanto, se $R_{0}<1$, então, da expresão (2.9) para $b_{0}$ e da última para $b_{1}$, temos que $b_{0}>0$ e $b_{1}>0$. Pode-se, assim, concluir que:

1. Se $R_{0}<1$, então $b_{0}>0$ e $b_{1}>0$, e não existe solução positiva.

2. Se $R_{0}>1$, então $b_{0}<0$, e, independente do sinal de $b_{1}$, existe uma única solução positiva.

Com respeito a pontos de equilíbrio, tem-se que $L_{2}=0$ sempre é solução de (2.8), e estável, se $R_{0}<1$, como foi mostrado em Teorema 2.1. Quando $R_{0}>1$, a solução trivial deixa de ser estável, e surge uma solução positiva, que é única.

Para a estabilidade da solução não trivial, temos o seguinte resultado:

Lema 2.1. Seja $P(\lambda)=\operatorname{det}(J-\lambda I)=\sum_{i=0}^{7} c_{7-i} \lambda^{i}$, onde $J$ é o Jacobiano formado pelas derivadas parciais em relação às variáveis de estados que estão no segundo membro de (2.5). Então o número de reprodutibilidade basal $R_{0}$, caso ele seja maior ou menor que um, determina a mudança de sinal em $\overline{c_{7}}$, onde $\overline{c_{7}}$ é a parte do coeficiente $c_{7}$ do polinômio $P(\lambda)$ que não depende explicitamente da fração de Lutzomyia infectados $L_{2}$. 
Demonstração. O termo independente de $\lambda$ da expressão det $(J)$, onde $J$ é calculada com as coordenadas do ponto de equilíbrio não trivial, é dado por

$$
P(0)=\operatorname{det}(J)=\mu_{1} \mu_{2} \mu_{3}\left(A_{1} L_{2}^{2}+A_{2} L_{2}+A_{3}\right),
$$

com os coeficientes $A_{1}, A_{2}$ e $A_{3}$ dados por

$$
\left\{\begin{aligned}
A_{1}= & -\left(\mu_{1}+\theta_{1}+\gamma_{1}\right)\left(\mu_{2}+\theta_{2}+\gamma_{2}\right)\left(\overline{\mu_{3}}+\beta_{1} H_{2}+\beta_{2} C_{2}\right) \alpha_{1} \alpha_{2} \\
A_{2}= & \left(\mu_{2}+\theta_{2}+\gamma_{2}\right) \alpha_{2} \\
& \times\left[\left(\mu_{1}+\theta_{1}\right) \overline{\mu_{1}}\left(\overline{\mu_{3}}+\beta_{1} H_{2}+\beta_{2} C_{2}\right)-\left(\mu_{1}+\theta_{1}\right) \alpha_{1} \beta_{1} H_{1} L_{1}\right] \\
& +\left(\mu_{2}+\theta_{2}\right)\left(\mu_{1}+\theta_{1}+\gamma_{1}\right)\left(\overline{\mu_{3}}+\beta_{1} H_{2}+\beta_{2} C_{2}\right) \alpha_{1} \\
A_{3}= & \left(\mu_{2}+\theta_{2}\right)\left(\mu_{1}+\theta_{1}\right) \\
& \times\left[-\overline{\mu_{1} \mu_{2}}\left(\overline{\mu_{3}}+\beta_{1} H_{2}+\beta_{2} C_{2}\right)+\alpha_{2} \beta_{2} \overline{\mu_{1}} C_{1} L_{1}+\alpha_{1} \beta_{1} \overline{\mu_{1}} H_{1} L_{1}\right] .
\end{aligned}\right.
$$

A expressão para $\beta_{1} H_{2}+\beta_{2} C_{2}$ resulta, usando as equações (2.5) e (2.6), em

$$
\beta_{1} H_{2}+\beta_{2} C_{2}=\frac{B_{1} L_{2}^{2}+B_{2} L_{2}}{D},
$$

onde $B_{1}, B_{2}$ e $D$ são

$$
\left\{\begin{array}{l}
B_{1}=\alpha_{1} \alpha_{2}\left[\beta_{1}\left(\mu_{1}+\theta_{1}\right)\left(\mu_{2}+\theta_{2}+\gamma_{2}\right)+\beta_{2}\left(\mu_{2}+\theta_{2}\right)\left(\mu_{1}+\theta_{1}+\gamma_{1}\right)\right] \\
B_{2}=\left(\mu_{1}+\theta_{1}\right)\left(\mu_{2}+\theta_{2}\right)\left[\alpha_{1} \beta_{1} \overline{\mu_{2}}+\alpha_{2} \beta_{2} \overline{\mu_{1}}\right] \\
D=D_{1} L_{2}^{2}+D_{2} L_{2}+D_{3}
\end{array}\right.
$$

com $D_{1}, D_{2}$ e $D_{3}$ dados por

$$
\left\{\begin{array}{l}
D_{1}=\left(\mu_{1}+\theta_{1}+\gamma_{1}\right)\left(\mu_{2}+\theta_{2}+\gamma_{2}\right) \alpha_{1} \alpha_{2} \\
D_{2}=\left(\mu_{1}+\theta_{1}\right) \overline{\mu_{1}}\left(\mu_{2}+\theta_{2}+\gamma_{2}\right) \alpha_{2}+\left(\mu_{2}+\theta_{2}\right) \overline{\mu_{2}}\left(\mu_{1}+\theta_{1}+\gamma_{1}\right) \alpha_{1} \\
D_{3}=\left(\mu_{1}+\theta_{1}\right)\left(\mu_{2}+\theta_{2}\right) \overline{\mu_{1} \mu_{2}}
\end{array}\right.
$$

Portanto, do termo que não depende de $L_{2}$ explicitamente, analisando $A_{3}$ e $D_{3}$, tem-se que

$$
\left.\overline{c_{7}}=-\left(\mu_{1}+\theta_{1}\right)^{2}\left(\mu_{2}+\theta_{2}\right)^{2}{\overline{\mu_{1}}}^{2} \bar{\mu}_{2}^{2} \frac{\alpha_{3}}{\mu_{1} \beta_{1}} H_{1} L_{1}-\frac{\alpha_{2} \beta_{2}}{\overline{\mu_{1} \mu_{3}}} C_{1} L_{1}\right) .
$$

Agora, se $R_{0}>1$, devemos ter $L_{2}>0$, o que implica em $0<L_{1}<1$. Além disso, $L_{2}>0$ também implica que $C_{2}>0$ e, portanto, $0<C_{1}<1$. Analogamente, $H_{2}>0$ e, consequentemente, $0<H_{1}<1$. Logo, verificamos que

$$
\overline{c_{7}}>-\left(\mu_{1}+\theta_{1}\right)^{2}\left(\mu_{2}+\theta_{2}\right)^{2}{\overline{\mu_{1}}}^{2}{\overline{\mu_{2}}}^{2} \overline{\mu_{3}}\left(1-\frac{\alpha_{1} \beta_{1}}{\overline{\mu_{1} \mu_{3}}}-\frac{\alpha_{2} \beta_{2}}{\overline{\mu_{2} \mu_{3}}}\right)>0,
$$

pois $\left(1-R_{0}\right)<0$. Se $R_{0}<1$, então $\overline{c_{7}}<0$.

\section{Observações:}

a) Na expressão do determinante dada pela equação (2.10), se colocarmos os valores das coordenadas $(1,0,0,1,0,0,1,0)$ do ponto de equilíbrio trivial $\overline{P_{e_{1}}}$, recuperase a expresão $-\mu_{1} \mu_{2} \mu_{3}\left(\mu_{1}+\theta_{1}\right)\left(\mu_{2}+\theta_{2}\right) a_{3}$. Assim, a estabilidade depende apenas do sinal de $a_{3}$, como se mostrou. 
b) O fato de ocorrer uma mudança do sinal conforme o número de reprodutibilidade basal seja maior ou menor que um, faz com que a estabilidade também mude com $R_{0}$, e possa valer para o caso não trivial. O Lema 2.1 pode ajudar nessa conclusão do ponto de vista teórico, segundo a conjectura feita em [3].

\section{Resultado Numérico}

O modelo será aplicado para descrever zonas endêmicas do Estado de São Paulo, por exemplo, a situação epidemiológica encontrada no Município de Araçatuba. Usando variação dos coeficientes de incidência no Estado de SP entre 2000 e 2003, que foi de 2,7 a 34,7, estima-se os coeficientes $\alpha_{i}$. A ocorrência da LV em seres humanos tem como fator de risco a ocorrência de LV em cães com prevalência superior a $2 \%$ com uma alta densidade de população canina [1]. Para condições iniciais a fim de simular o sistema dinâmico $(2.1)$, usamos, para cães, $C_{1}=0,98 ; C_{2}=0,02$ e $C_{3}=0$; para homens, $H_{1}=1 ; H_{2}=0$ e $H_{3}=0$, enquanto para o vetor $L u$., $L_{1}=0,99$ e $L_{2}=0,01$.

Os valores dos parâmetros utilizados para obter o desenvolvimento no tempo das variáveis de estado são dados na Tabela 1. Quase todos os valores são obtidos do trabalho de Yang e Ferreira [8], segundo as referências por eles citadas, em que algumas adaptações foram feitas para o caso do vetor $L u$.

\begin{tabular}{lccc}
\hline Parâmetro & Valor & Parâmetro & Valor \\
\hline$\mu_{1}^{-1}$ & 52,5 & $\mu_{1}^{\prime}$ & 0,075 \\
$\mu_{2}^{-1}$ & 2,3 & $\mu_{2}^{\prime}$ & 0,0037 \\
$\mu_{3}^{-1}$ & 0,08 & $\left(\mu_{3}^{\prime}\right)^{-1}$ & 0,0016 \\
$\gamma_{1}^{-1}$ & $\frac{12}{7}$ & $\gamma_{2}^{-1}$ & 5 \\
$\alpha_{1}$ & 0,0347 & $\alpha_{2}$ & 12,4 \\
$\beta_{1}$ & 0,5 & $\beta_{2}$ & 5,4 \\
$\theta_{1}$ & 0,05 & $\theta_{2}$ & 0,01 \\
\hline
\end{tabular}

Tabela 1: Valores dos parâmetros de modelo usados na simulação, dados em dias $^{-1}$. Os valores são aqueles encontrados na literatura.

As simulações numéricas do sistema dinâmico (2.1) foram obtidas usando rotina ode 45, para o Problema do Valor Inicial do tipo não stiff (programa de MATLAB ${ }^{\circledR}$ ). A Figura 1 mostra uma simulação para o caso em que o número de reprodutibilidade basal global é $R_{0}=R_{0}^{C}+R_{0}^{H}=5,5938$, onde as contribuições individuais são $R_{0}^{C}=5,5924$ e $R_{0}^{H}=0,0014$. Como os cães são sentinelas, escolheu-se uma contribuição muito maior por parte de cães que hospedeiro humano na transmissão de Leishmaniose. Note que o número de reprodutibilidade basal com respeito à transmissão entre homens, $R_{0}^{H}$, é muito menor que o correspondente ao cão, $R_{0}^{C}$, o que permite a incidência baixa nos homens graças à manutenção da transmissão entre cães em nível elevada. Assim, ocasionalmente, surgem casos de Leishmaniose humana. 

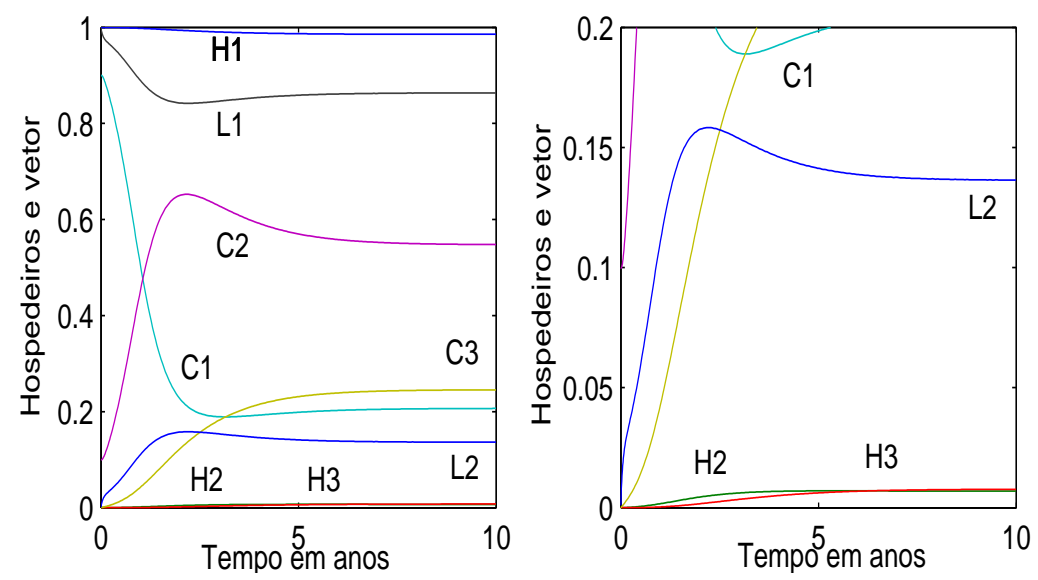

Figura 1: Dinâmica da transmissão da Leishmaniose. Resultados numéricos com dados de zona endêmica do Estado de São Paulo, Município de Araçatuba. Observase que o nível endêmico persiste elevado em cães, mesmo para baixo nível no vetor $L u$. A figura da direita mostra detalhes (diminuindo o valor máximo de prevalência) da mesma situação da figura à esquerda. As curvas dos compartimentos $\mathrm{H}_{2}$ e $\mathrm{H}_{3}$ são aquelas próximas do eixo horizontal (figura à esquerda), e a curva de $H_{2}$ é aquela que alcança a assíntota mais rapidamente (figura à direita). Os valores dos parâmetros do modelo resultaram em $R_{0}=R_{0}^{C}+R_{0}^{H}=5$, 5938, onde $R_{0}^{C}=5,5924$ e $R_{0}^{H}=0,0014$.

\section{Conclusão}

Desenvolveu-se um modelo matemático para descrever a transmissão de Leishmaniose considerando-se dois hospedeiros vertebrados e um invertebrado. O modelo foi analisado em seus estados de equilíbro. Derivou-se a expressão para o número de reprodutibilidade basal com seus componentes que fornecem contribuições efetivas de cada hospedeiro. As simulações mostram ocorrência de Leishmaniose no hospedeiro ocasional, que é o homem, como conseqüência da grande contribuição dos cães ao número de reprodutibilidade. Além disso, as simulações mostram, também, que os níveis endêmicos nos vertebrados persistem mesmo que a prevalência no vetor $L u$. seja baixa. Isto poderia explicar os resultados negativos de muitas pesquisas que procuram o protozoário no estômago dissecado de exemplares de $L u$. capturados.

Quando o ser humano interfere no habitat em que está inserido, sem os devidos cuidados, pode sofrer conseqüências desta ação. O vetor transmissor de Leishmaniose pode se estabelecer em locais próximos ao homem trazendo, consigo, a doença antes circunscrita na região da mata. Como fator de risco, encontram-se os cães que, mantendo a doença em níveis endêmicos na sua população, pode ocasionar a transmissão entre os homens. Os resultados deste trabalho alertam para este risco, mostrando que cuidadosas atenções devem ser dadas para regiões próximas da mata para não tornar Leishmaniose endêmica nas grandes cidades. Uma forma 
de controle de Leishmaniose humana é o manejo responsável e cuidadoso das matas e florestas que circundam povoados. Outra forma de evitar a transmissão entre homens em regiões endêmicas consiste no controle da doença nos cães, em especial aqueles abandonados.

\section{Agradecimentos}

Os autores agradecem as sugestões dos revisores anônimos que contribuíram substancialmente no aprimoramento desse trabalho.

Abstract. This work describes the transmission of Leishmaniasis considering compartmental model. This model takes into account three hots (two vertebrates and one invertebrate). The analytical expression for the basic reproduction number is derived through stability analysis. We made simulation with data obtained from endemic zone in the State of São Paulo.

\section{Referências}

[1] V.L.F. Camargo-Neves, A Leishmaniose Visceral Americana no Estado de São Paulo: Situação Atual, Boletim Epidemiológico Paulista, 6 (2004), s/n (http://www.cve.saude.sp.gov.br/agencia/bepa6_lva.htm).

[2] L. Edelstein-Keshet, "Mathematical Models in Biology", McGraw-Hill, Inc., New York, 1988.

[3] M.B.F. Leite, R.C. Bassanezi, H.M. Yang, The Basic Reproduction Ratio for Model of Directly Transmitted Infections Considering The Virus Chargue and Immunological Response, IMA J Math Appl Med Biol, 17, No. 3 (2000), 15-31.

[4] D.H. Molyneux, R.W. Ashford, "The Biology of Tripanosoma and Leishmania. Parasites of Man and Domestic Animals", International Publications Service, 1983.

[5] J.D. Murray, "Mathematical Biology I: An Introduction", Ed. Springer, 1993.

[6] D.P. Neves, "Parasitologia Humana", 10ª Ed., Atheneu, 2000.

[7] Site WHO. TDR. http://www.who.int/tdr/, acesso em Março/2005.

[8] H.M.Yang, M. Ferreira, Malaria Transmission Model for Different Levels of Acquired Immunity and Temperature-dependente Parameters (Vector), Rev. Saúde Pública, 34, No. 3 (2000), 223-231. 